\title{
Reliability of Heuristic Evaluation during Usability Analysis
}

Thomas J. Smith and Cindy Kheng

University of Minnesota, School of Kinesiology

\section{Abstract}

Scientific reliability --- the degree to which a research method produces stable and consistent results -2 tepresents a major linchpin of tenable scientific research. A number of different studies support the conclusion that usability testing of software report augments this conclusion with findings that heuristic evaluation, the present major pillar of usability analysis, also lacks reliability. We conclude that a key priority of "usability science" should be to initiate a systematic program of inquiry to investigate the degree to which this term has meaning.

\section{Objective}

Analyze the reliability of heuristic rankings of different design features attributed to selected social networking sites.

\section{Background}

A number of different studies support the conclusion that usability testing of software lacks scientific reliability. As stated by Jacobbsenn et. al. [2] "Both selection of usability

Factors that affect the probability that a problem will be detected by usability test - Number of problems contained in a given interface

Difficulty in detecting a given problem

This study extends prior research on usability testing by exploring the reliability of heuristic evaluation, the second scientific pillar of usability analysis.

\section{Method}

Student teams enrolled in a human-centered design course were tasked with members and members of recruited survey groups used the survey to numerically rate the quality of a series of heuristics relevant to different design features of the sites.

These heuristic quality rating data then were analyzed using correlation analysis and Krippendorff's alpha (K-alpha) to assess the reliability of the results, based on evaluation of the varlance in tean versus survey group member ratings for each heuristic.

\section{Results}

I. Correlation Analysis

Table 1 summarizes quality rating results for a series of different heuristics for four different social networking sites. Average Team and Survey Member Ratings are based quality ratings by $=23 ; r=0.37$ ) is statistically non-significant.
Table 1. Heuristic Evaluation Based on Quality Ratings of Selected Heuristics

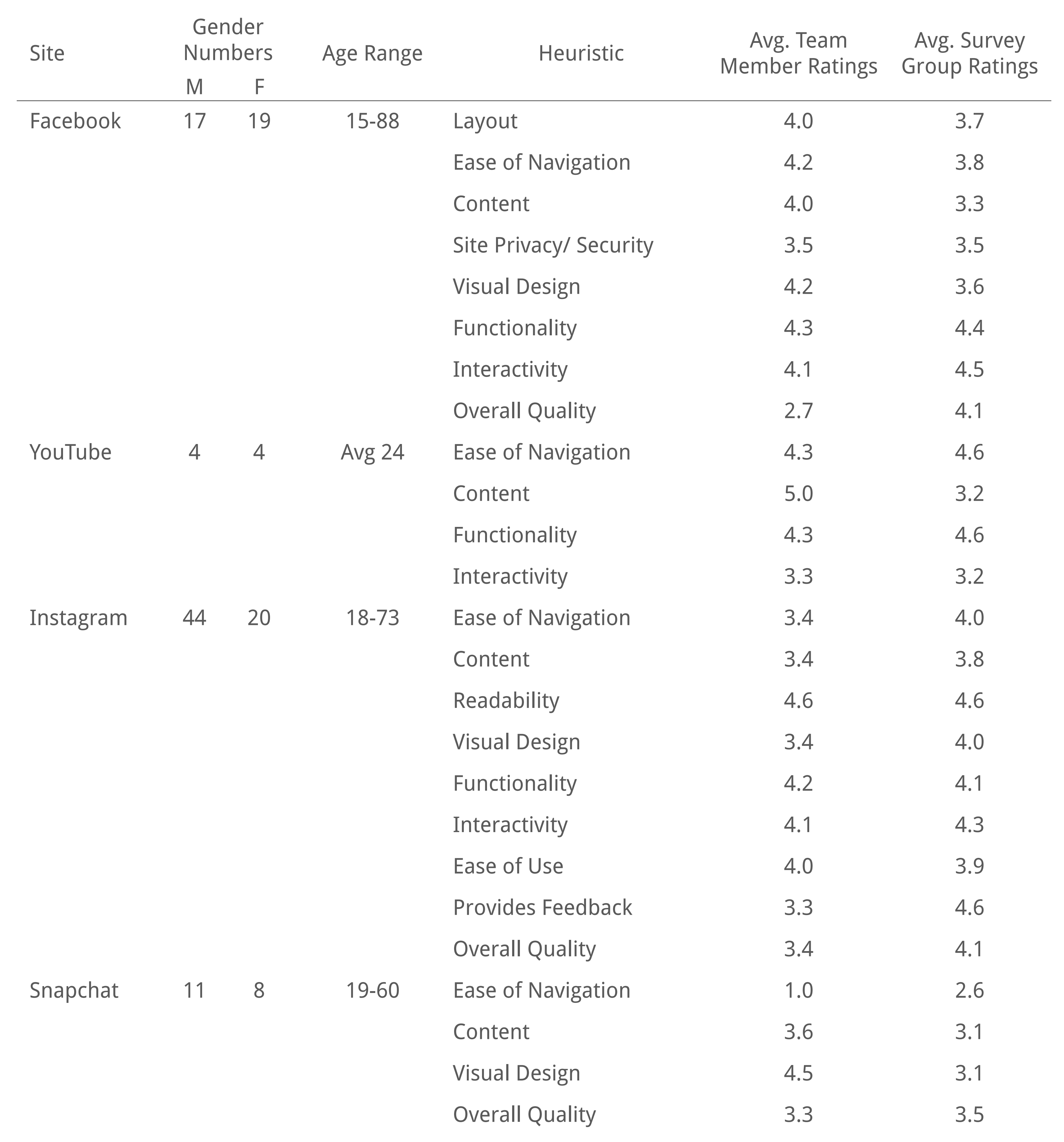

Figure 1. Scatter plot of paired average heuristic quality ratings

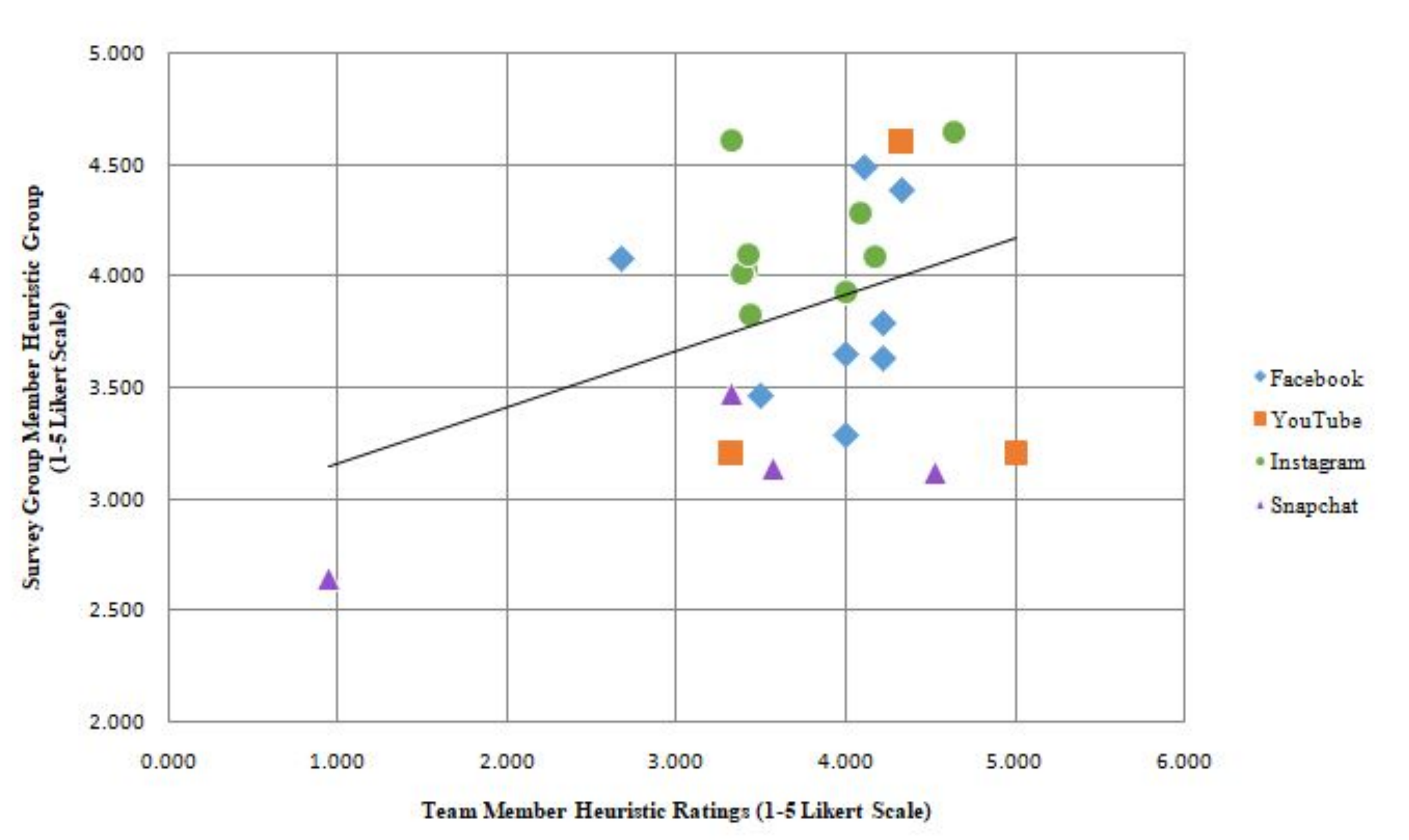

Table 1. Individual site data and combined data K-alpha

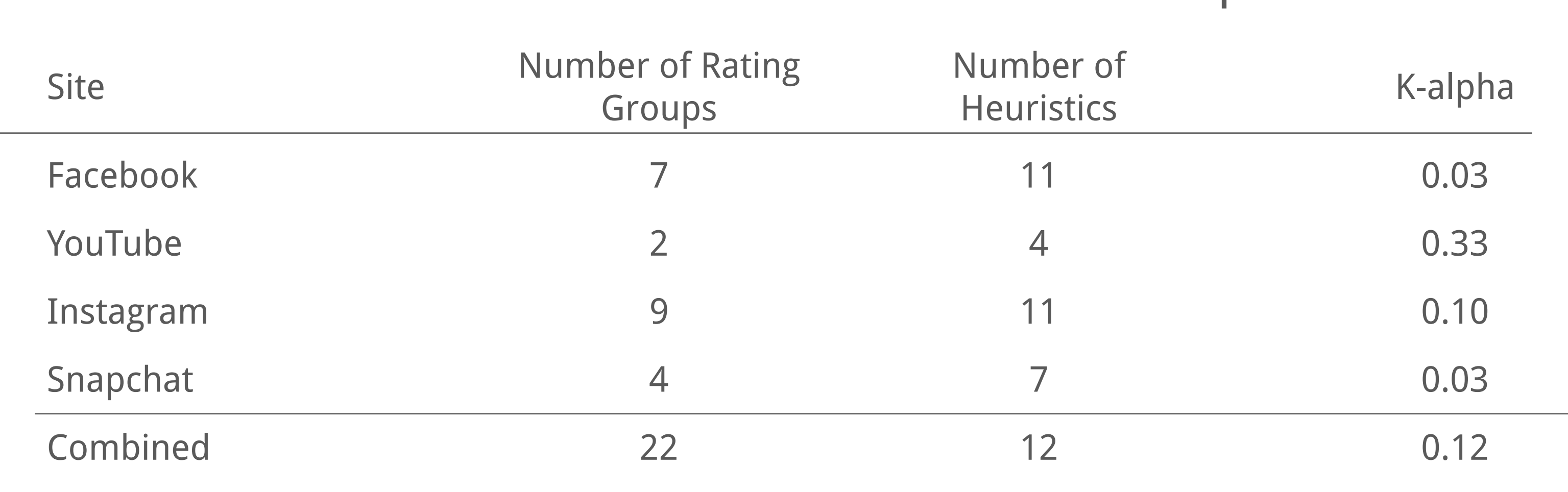

\section{Results (continued)}

II. Krippendorff's Alpha

Table 1 lists K-alpha values for each social networking site, based on inter-rater (group) agreement on quality ratings for each heuristic, plus the value calculated for al

The finding from these two analytical approaches shows that heuristic evaluation by independent groups of raters lacks reliability.

\section{Conclusion \& Discussion}

The analysis in this report raises the following major questions and conclusions: . Does a lack of reliability in ratings of heuristic quality represent a reasonable, or eve 2. What is an acceptable level of variability in judgments of heuristic quality for a given interface design?

3. Given that prior research on usability testing coupled with the evaluation of heuristic quality ratings presented in this report, both point to a lack of reliability in these empirical pillars of usability analysis, a reasonable conclusion is that the scientific validity of usability analysis is open to question.

Given the latter conclusion, a key priority of "usability science" should be to initiate a systematic program of inquiry to investigate the degree to which this term has meaning

\section{References}

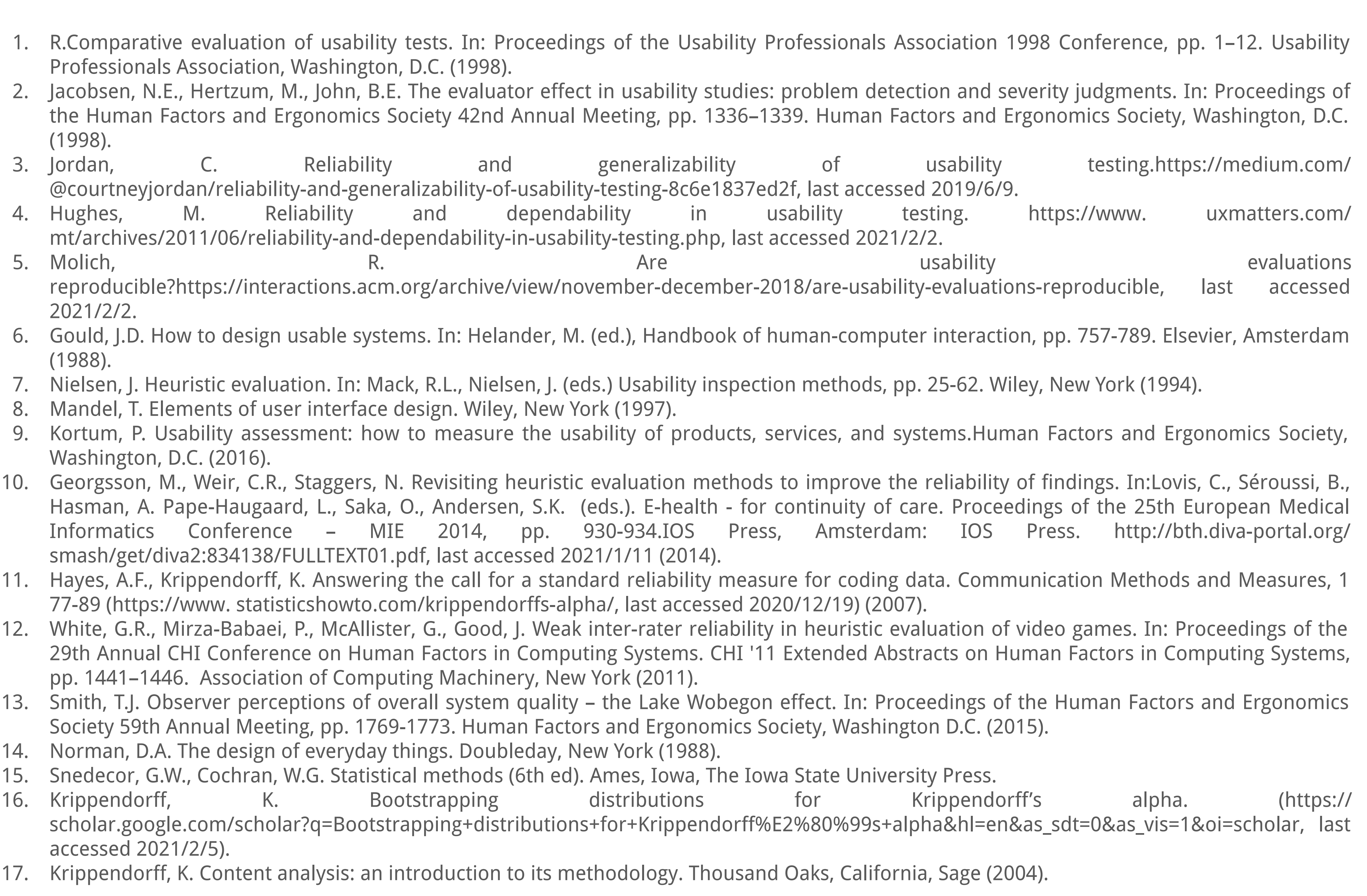




\section{和}

\section{Reliability of Heuristic Evaluation during Usability Analysis}

Thomas J. Smith and Cindy Kheng

University of Minnesota, School of Kinesiology

\section{Abstract}

Scientific reliability --- the degree to which a research method produces stable and consistent results --- represents a major linchpin of tenable scientific research. A number of different studies support the conclusion that usability testing of software report augments this conclusion with findings that heuristic evaluation the present major pillar of usability analysis, also lacks reliability. We conclude that a key priority of "usability science" should be to initiate a systematic program of inquiry to investigate the degree to which this term has meaning.

\section{Objective}

Analyze the reliability of heuristic rankings of different design features attributed to selected social networking sites.

\section{Background}

A number of past studies support the conclusion that usability testing of software lacks scient section of the most severe probems a "Bubjett to considerable individua

Factors that affect the probability that a problem will be detected by usability tes: - Number of problems contaned in a given interface

Difficulty in detecting a given problem

usability of interface

This study extends prior research on usability testing by exploring the reliability of heuristic evaluation, the second scientific pillar of usability analysis.

\section{Method}

Student teams enrolled in a human-centered design course carried our usability analysis of selected social networking sitess, using a perceptual survey. For this purpose, team members and recruited survey group members numerically rated the quality of a series of heuristics applicable to the deion of these sites.

The heuristic data then were analyzed using correlation analysis and Krippendorff's alpha. Reliability of the results was ascertained by evaluating the variance in ratings by team versus survey group members for each heuristic.

\section{Results}

I. Correlation Analysis

Table 1 summarizes quality rating results for a series of different heuristics for four different social networking sites. Average Team and survey Member Ratings are based quality ratings by team members vs. survey group members from Table 1 . The regression ( $\mathrm{DOF}=23$, and $r=0.37$ ) is statistically non-significant.

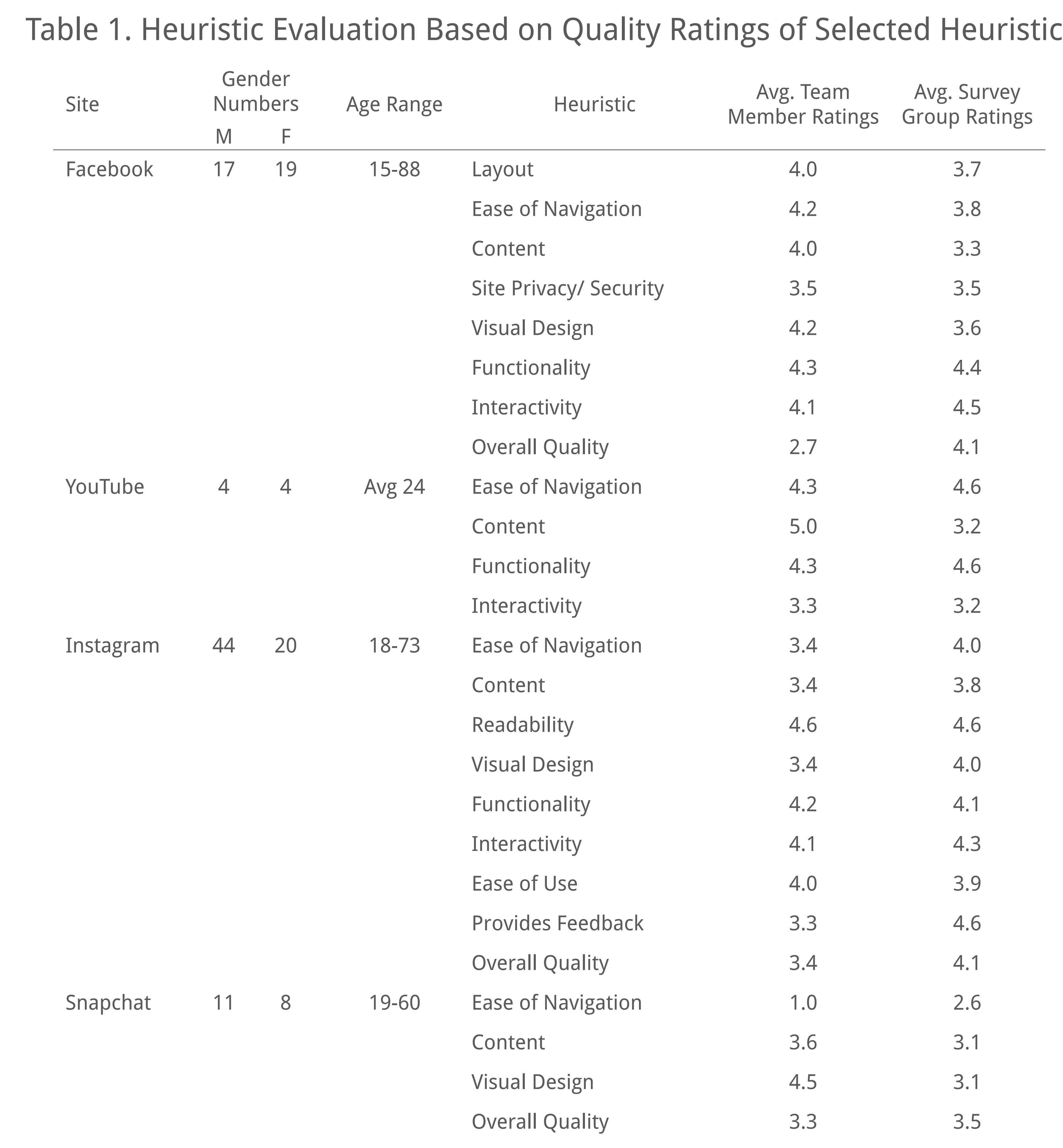

Figure 1. Scatter plot of paired average heuristic quality ratings

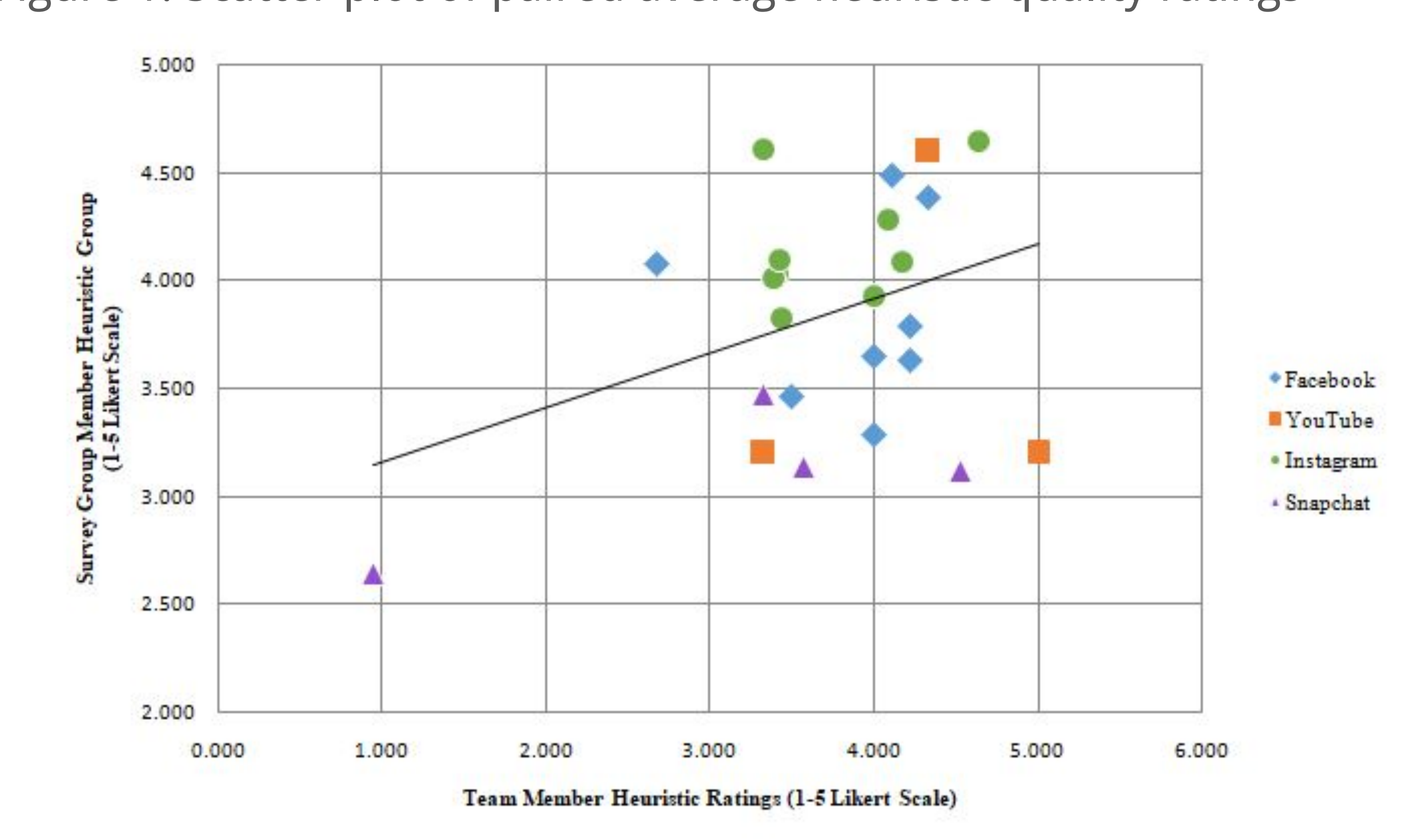

Table 2. Individual site data and combined data K-alpha

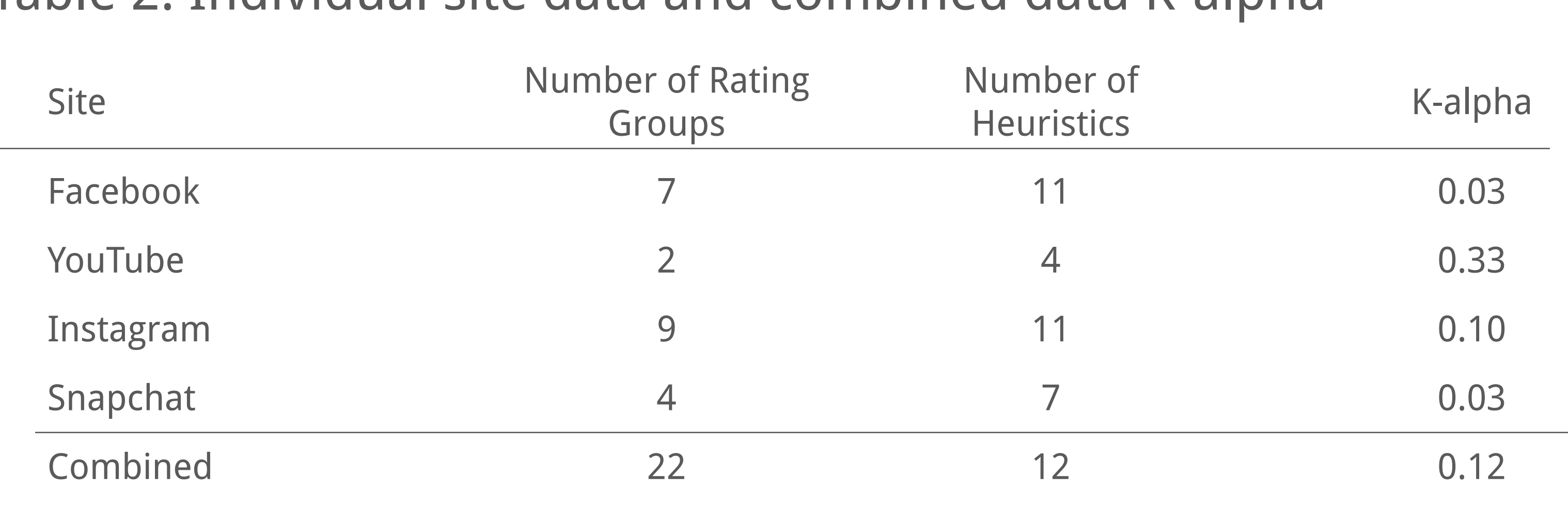

\section{Results}

II. Krippendorff's Alpha

Figure 2 lists Kalpha values of each social networking site, based on inter-rater (group) agreement on quality ratings for each heuristic, plus the value calculated for all rating

The finding from these two analytical approaches shows that heuristic evaluation by independent groups of raters lacks reliability.

\section{Conclusion \& Discussion}

The analysis in this report addresses the following major questions and conclusions: . Does a lack of reliability in ratings of heuristic quality represent a reasonable, or eve . What is a expectab?

(evel variability in judgments of heuristic quality for a given

3. Given that prior research on usability testing, coupled with the evaluation of heuristic quality ratings presented in this report, both point to a lack of reliability in these empirical pillars of usability analysis, a reasonable conclusion is that the scientific validity of usability analysis is open to question.

Given this conclusion, a key priority of "usability science" should be to initiate a systematic program of inquiry to investigate the degree to which this term has meaning.

\section{References}

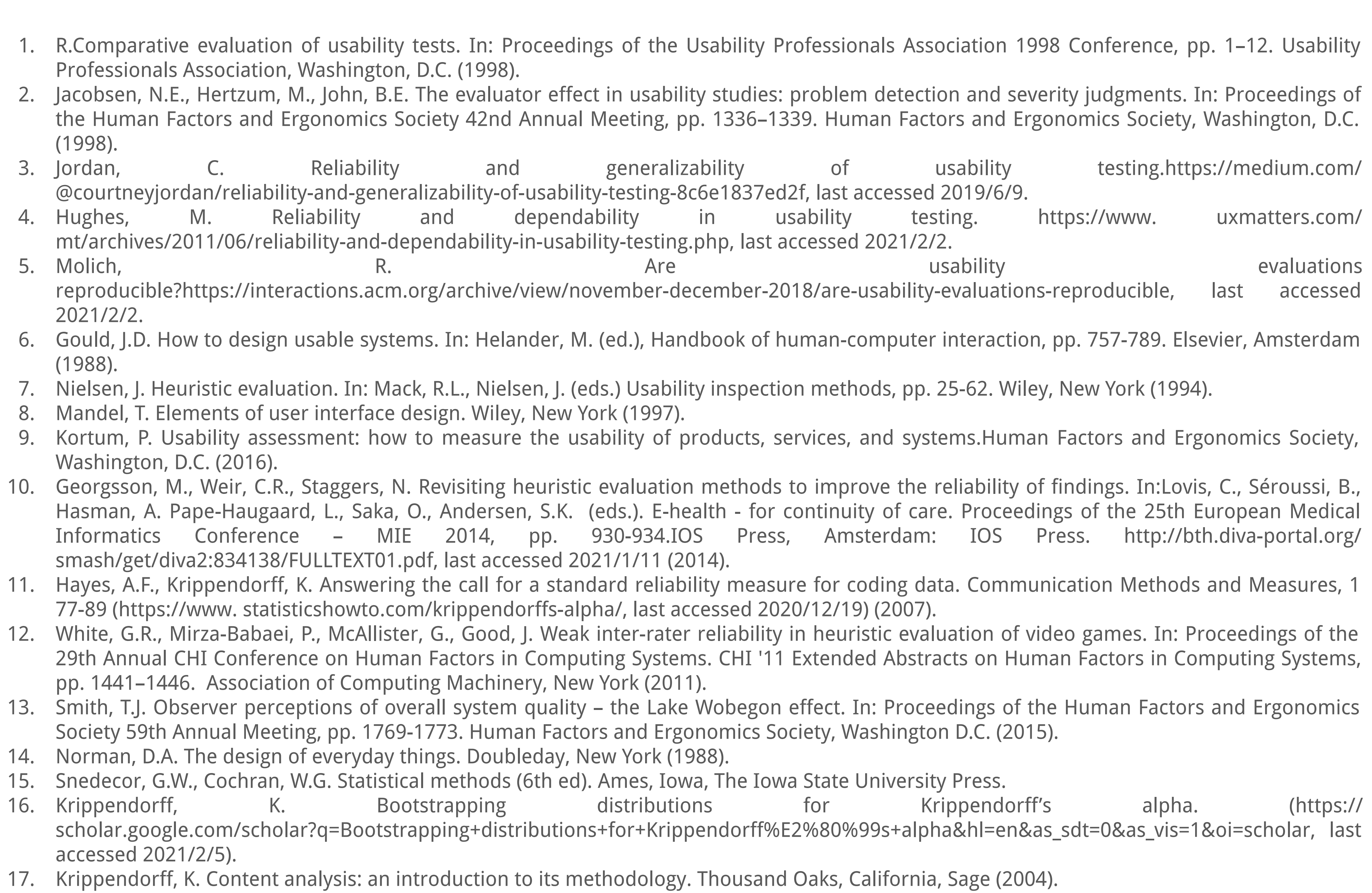

School of Kinesiology 